\title{
Nonlocal Theory to Analyse Nanotube Structures under Tension
}

\author{
Hamidreza Yazdani Sarvestani and Ali Naghashpour \\ Concordia Centre for Composites (CONCOM), Department of Mechanical and Industrial Engineering, Concordia University, \\ Montreal, Canada H3G 1 M8 \\ Correspondence should be addressed to Hamidreza Yazdani Sarvestani; h_yazd@encs.concordia.ca
}

Received 28 January 2015; Accepted 15 April 2015

Academic Editor: Victor M. Castaño

Copyright (c) $2015 \mathrm{H}$. Yazdani Sarvestani and A. Naghashpour. This is an open access article distributed under the Creative Commons Attribution License, which permits unrestricted use, distribution, and reproduction in any medium, provided the original work is properly cited.

\begin{abstract}
Because nanocomposites have found augmented use in many industries, the analytical solutions are required to be developed. This paper presents the development of a new analytical method for studying nanotube structures under tension using layer-wise and Eringen theories. Two opposite ends of tubes are subjected to normal forces. Nonlocal governing differential equations are derived and presented. The theoretical developments determine the effect of the geometric and nonlocal constitutive relations for singlewalled nanotubes (SWNTs), double-walled nanotubes (DWNTs), and multiwalled nanotubes (MWNTs) under tension loading. It is observed that all displacement components increase with the increase in the nonlocal parameter.
\end{abstract}

\section{Introduction}

Carbon nanotubes (CNTs) have been used as multifunctional materials while analytical and numerical analyses are required to be performed for CNT structures. Naghashpour and Hoa $[1,2]$ dispersed CNTs into polymer composites to produce multifunctional polymer composites. Significant amount of experimental work on the use of CNTs in composites is being conducted while analytical and numerical analyses are required to be performed to obtain the properties and behaviors of nanocomposites.

The vast majority of structural theories are derived using the constitutive assumptions that the stress at a point depends only on the strain at that point. Many investigations were made on the development of analytical solutions to analyse the composite structures $[3,4]$ while it remains to be observed whether these solutions can be applied to make analyses for nanostructures. Continuum based analyses have been generally utilized for the formulation of different nanostructures. Two main reasons are mentioned. One is experimental investigations in nanoscale that are difficult. The other is molecular dynamic simulations being highly computationally expensive for nanostructures. Over the past decade, some researchers have applied classical continuum mechanics such as EulerBernoulli theory, Timoshenko beam theory, and Kirchhoff's plate theory to predict the behavior of nanostructures $[5,6]$.
The nonlocal constitutive behaviour developed by Eringen [7-10] is based on the hypothesis that the stress at a point is a function of strains at all points in the continuum. The nonlocal elasticity theory was used for linear isotropic nonlocal solids by Eringen and Kim [11], in such a way that the nonlocal theory differs from the local theory in the stressstrain constitutive relations. Since the classical continuum elasticity is a scale-free theory, the use of classical continuum models may be not able to be relied on in the analysis of structural elements in nanoscale such as CNTs and graphene sheets. There are different modified classical continuum theories which capture size effects such as couple stress theory [12], strain gradient elasticity theory [13], modified couple stress theory $[14,15]$, and nonlocal elasticity theory [7-11]. Among all size-dependent theories, the nonlocal elasticity theory has been frequently used in the theoretical investigations of structures at small scale [16-21]. Heireche et al. [18] analysed the wave propagation in CNTs by developing a single-elastic beam model using nonlocal elasticity. By considering small-scale effect, the buckling behavior of nanoscale circular plates under uniform radial compression was investigated [20]. Mohammadi et al. [21] investigated the free vibration behavior of circular and annular graphene sheet by utilizing elasticity theory. They also derived the governing equations for single-layered graphene sheets (SLGS). Reddy [22-24] used different beam theories including those of 


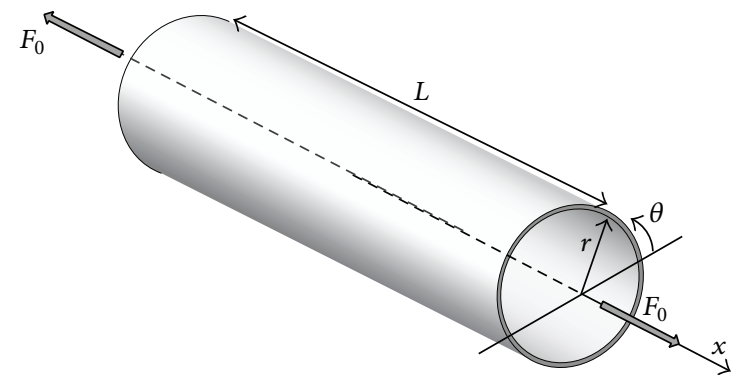

FIGURE 1: Schematic sketch of SWNT under axial force and the location of coordinate axes.

Euler-Bernoulli, Timoshenko, and Levinson to analyze bending, buckling, and vibration of nonlocal beams. In his study, different displacement functions were selected in the first step and then all steps were redone when deriving beam equilibrium and equations of motion. Recently, a finite element method was developed for a nonlocal Timoshenko beam model [25]. The model was based on the key idea that nonlocal effects include long-range volume forces and moments exchanged by nonadjacent beam segments.

It is found from the presented works that there is a lack of research for developing a modeling method that analyzes the mechanical response of nanotubes under extension. Even though a lot of researchers have considered nanotube as a sheet for their analysing, a tube structure is considered here to develop a displacement based theory to analysis of nanotubes. In the current work, we are studying the deformation of nanotube structures under axial forces based on the nonlocal elasticity theory in which the small-scale effects are taken into account. The displacement based governing equations of layer-wise theory are developed. Then, the nonlocal constitutive relations are used to express the stress resultants in terms of the generalized displacements. Finally, the equations are solved for specified axial force. The formulations show that the nonlocal parameter has prominent effect on the behavior of nanotube structures. It is anticipated that the results of the present work would be helpful for designing composite structures using SWNT or/and DWNT or/and MWNT. In addition, since direct experimental measurements are impractical due to the very small size of CNTs, the developed theoretical approach provides good alternative.

\section{Theoretical Formulation}

2.1. Displacement Field. SWNT, DWNT, and MWNT with mean radius $R$ and thickness $h$ are subjected to a torque as shown in Figures 1, 2, and 3, respectively. The cylindrical coordinates $(x, \theta, r)$ are placed at the middle wall of the nanotubes so that $x$ and $r$ are the axial and radial coordinate, respectively. The integration of the appropriate linear straindisplacement relations of elasticity within cylindrical coordinate system will yield the following displacement field for the $k$ th wall:

$$
\begin{aligned}
u_{1}^{(k)}(x, \theta, r)= & x r\left(C_{5}^{(k)} \cos \theta+C_{4}^{(k)} \sin \theta\right)+C_{6}^{(k)} x \\
& +u^{(k)}(\theta, r),
\end{aligned}
$$

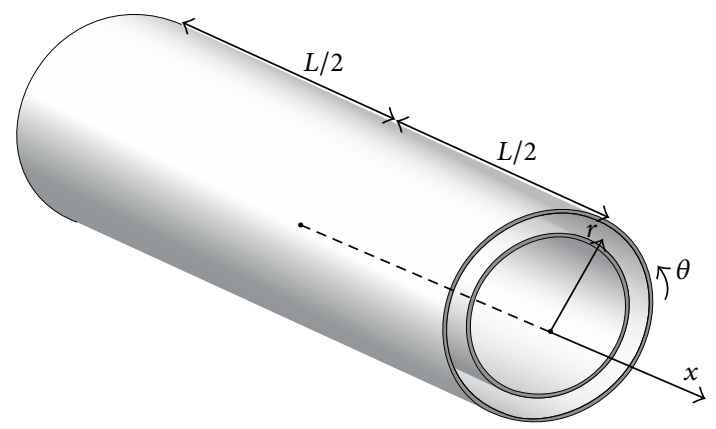

FIGURE 2: Schematic sketch of DWNT and the location of coordinate axes.

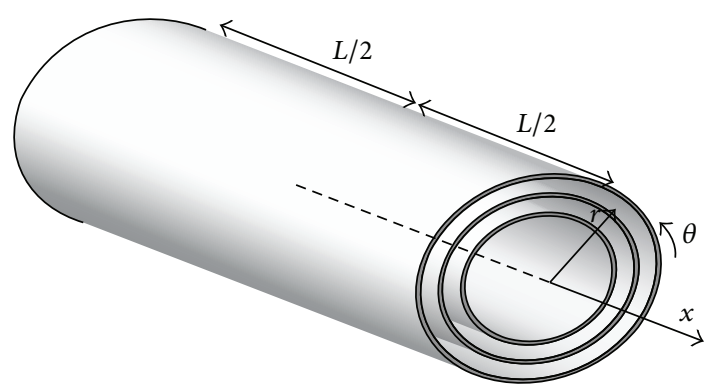

FIGURE 3: Schematic sketch of MWNT and the location of coordinate axes.

$$
\begin{aligned}
u_{2}^{(k)}(x, \theta, r)= & x\left(C_{1}^{(k)} \cos \theta-C_{2}^{(k)} \sin \theta-C_{3}^{(k)} r\right) \\
& -\frac{1}{2} x^{2}\left(C_{4}^{(k)} \cos \theta-C_{5}^{(k)} \sin \theta\right) \\
& +v^{(k)}(\theta, r) \\
u_{3}^{(k)}(x, \theta, r)= & x\left(C_{1}^{(k)} \sin \theta+C_{2}^{(k)} \cos \theta\right) \\
& -\frac{1}{2} x^{2}\left(C_{5}^{(k)} \cos \theta+C_{4}^{(k)} \sin \theta\right) \\
& +w^{(k)}(\theta, r),
\end{aligned}
$$

where $u_{1}^{(k)}(x, \theta, r), u_{2}^{(k)}(x, \theta, r)$, and $u_{3}^{(k)}(x, \theta, r)$ represent the displacement components in the $x, \theta$, and $r$ directions, respectively, of a material point located at $(x, \theta, r)$ in the $k$ th wall of the nanotube in Figures 1, 2, and 3. In order to satisfy the interfacial continuities of the displacement components, it is necessary for the integration constants appearing in (1a), (1b), and (1c) to be the same for all walls. Thus, relations (la), (1b), and (lc) are presented as

$$
\begin{aligned}
u_{1}^{(k)}(x, \theta, r)= & x r\left(C_{5} \cos \theta+C_{4} \sin \theta\right)+C_{6} x \\
& +u^{(k)}(\theta, r)
\end{aligned}
$$




$$
\begin{aligned}
u_{2}^{(k)}(x, \theta, r)= & x\left(C_{1} \cos \theta-C_{2} \sin \theta-C_{3} r\right) \\
& -\frac{1}{2} x^{2}\left(C_{4} \cos \theta-C_{5} \sin \theta\right) \\
& +v^{(k)}(\theta, r), \\
u_{3}^{(k)}(x, \theta, r)= & x\left(C_{1} \sin \theta+C_{2} \cos \theta\right) \\
& -\frac{1}{2} x^{2}\left(C_{5} \cos \theta+C_{4} \sin \theta\right) \\
& +w^{(k)}(\theta, r) .
\end{aligned}
$$

Moreover, in $(2 \mathrm{a}) u^{(k)}(\theta, r)$ can be replaced by $-C_{1} r \sin \theta+$ $u^{(k)}(\theta, r)$; it can be verified that the terms involving $C_{1}$ in $(2 \mathrm{a}),(2 \mathrm{~b})$, and $(2 \mathrm{c})$ correspond to an infinitesimal rigidbody rotation. These terms will, therefore, be ignored in the following developments since they will generate no strain. Similarly, it can be readily shown that the terms involving $C_{2}$ must also be eliminated since they represent another rigidbody rotation of the tube. Furthermore, as long as the loading conditions at the two ends of the nanotube are identical, the constant $C_{4}$ must vanish in order to satisfy the symmetry condition in deformation $u_{3}^{(k)}(x, \theta, r)=u_{3}^{(k)}(-x,-\theta, r)$. It is thus concluded that the most general form of the displacement field for the $k$ th wall of a nanotube is given as

$$
\begin{aligned}
& u_{1}^{(k)}(x, \theta, r)=C_{5} x r \cos \theta+C_{6} x+u^{(k)}(\theta, r), \\
& u_{2}^{(k)}(x, \theta, r)=-C_{3} r x+\frac{1}{2} C_{5} x^{2} \sin \theta+v^{(k)}(\theta, r), \\
& u_{3}^{(k)}(x, \theta, r)=-\frac{1}{2} C_{5} x^{2} \cos \theta+w^{(k)}(\theta, r) .
\end{aligned}
$$

2.2. Layer-Wise Theory (LWT). Various theories such as the equivalent single-layer theories are unable to precisely represent the local phenomena in laminated composites. But then, the LWTs, which allow each layer of the laminate to act like a real three-dimensional layer, are able to present excellent results in definition of the localized phenomena. In LWT, the displacement components of a generic point in the laminate are conveniently given as

$$
\begin{aligned}
& u_{1}(x, \theta, z)=u_{k}(x, \theta) \Phi_{k}(z), \\
& u_{2}(x, \theta, z)=v_{k}(x, \theta) \Phi_{k}(z), \\
& u_{3}(x, \theta, z)=w_{k}(x, \theta) \Phi_{k}(z)
\end{aligned}
$$

$$
(k=1,2, \ldots, N+1),
$$

with $k$, here and in what follows, being a dummy index implying summation of terms from $k=1$ to $k=N+1$. In (4a), (4b), and (4c), $u_{1}, u_{2}$, and $u_{3}$ denote the displacement components in the $x, \theta$, and $r$ directions, respectively. Also, $u_{k}(x, \theta), v_{k}(x, \theta)$, and $w_{k}(x, \theta)$ represent the displacements of the points initially located on the $k$ th wall of the nanotube in the $x, \theta$, and $r$ directions, respectively. Furthermore, $\Phi_{k}(z)$ is the global Lagrangian interpolation function that is used for the discretization of the displacement through thickness. The linear global interpolation function is defined as

$$
\begin{aligned}
& \phi_{k}(z)= \begin{cases}0 & z \leq z_{k-1} \\
\psi_{k-1}^{2}(z) & z_{k-1} \leq z \leq z_{k} \\
\psi_{k}^{1}(z) & z_{k} \leq z \leq z_{k+1} \\
0 & z \geq z_{k+1}\end{cases} \\
& (k=1,2, \ldots, N+1),
\end{aligned}
$$

where $\psi_{k}^{j}(j=1,2)$ are the local Lagrangian linear interpolation functions within the $k$ th wall which are defined as

$$
\begin{aligned}
& \psi_{k}^{1}(z)=\frac{1}{h_{k}}\left(z_{k+1}-z\right), \\
& \psi_{k}^{2}(z)=\frac{1}{h_{k}}\left(z-z_{k}\right),
\end{aligned}
$$

where $h_{k}$ is the thickness of the $k$ th wall.

Based on the reduced elasticity displacement field in (3a), (3b), and (3c), the LWT displacement field in (4a), (4b), and (4c) is rewritten as

$$
\begin{aligned}
u_{1}^{(k)}(x, \theta, z)= & C_{5} x(R+z) \cos \theta+C_{6} x \\
& +U_{k}(\theta) \Phi_{k}(z), \\
u_{2}^{(k)}(x, \theta, z)= & -C_{3}(R+z) x+\frac{1}{2} C_{5} x^{2} \sin \theta \\
& +V_{k}(\theta) \Phi_{k}(z), \\
u_{3}^{(k)}(x, \theta, z)= & -\frac{1}{2} C_{5} x^{2} \cos \theta+W_{k}(\theta) \Phi_{k}(z) .
\end{aligned}
$$

By introducing $r=R+z$ and considering thin-shell assumption (i.e., $1+z / R \approx 1$ ), the strain-displacement relations are given as

$$
\begin{aligned}
\varepsilon_{x} & =\frac{\partial u_{1}}{\partial x}, \\
\varepsilon_{\theta} & =\frac{1}{R} \frac{\partial u_{2}}{\partial \theta}+\frac{u_{3}}{R}, \\
\gamma_{x \theta} & =\frac{\partial u_{2}}{\partial x}+\frac{1}{R} \frac{\partial u_{1}}{\partial \theta}, \\
\gamma_{\theta z} & =\frac{1}{R} \frac{\partial u_{3}}{\partial \theta}+\frac{\partial u_{2}}{\partial z}-\frac{u_{2}}{R}, \\
\gamma_{x z} & =\frac{\partial u_{3}}{\partial x}+\frac{\partial u_{1}}{\partial z}, \\
\varepsilon_{z} & =\frac{\partial u_{3}}{\partial z} .
\end{aligned}
$$


Substitution of (7a), (7b), and (7c) into the strain-displacement relations (8) yields the following results:

$$
\begin{aligned}
& \varepsilon_{x}=(R+z) C_{5} \cos \theta+C_{6}, \\
& \varepsilon_{\theta}=\left(V_{k}^{\prime}+W_{k}\right) \frac{\Phi_{k}}{R}, \\
& \varepsilon_{z}=W_{k} \Phi_{k}^{\prime}, \\
& \gamma_{\theta z}=\left(W_{k}^{\prime}-V_{k}\right) \frac{\Phi_{k}}{R}+V_{k} \Phi_{k}^{\prime}, \\
& \gamma_{x z}=U_{k} \Phi_{k}^{\prime}, \\
& \gamma_{x \theta}=-C_{3}(R+z)+U_{k}^{\prime} \frac{\Phi_{k}}{R} .
\end{aligned}
$$

In (9) and what follows, a prime indicates an ordinary differentiation with respect to an appropriate variable (i.e., either $\theta$ or $z$ ). The equilibrium equations of a straight tube with $N$ numerical walls are obtained by employing (9) in the principle of minimum total potential energy. The results are, in general, $3(N+1)$ local equilibrium equations corresponding to $3(N+1)$ unknown functions $U_{k}, V_{k}$, and $W_{k}$ and three global equilibrium equations associated with the three parameters $C_{3}, C_{5}$, and $C_{6}$.

According to the principle of minimum total potential energy at the equilibrium configuration of a body the variation of the total potential energy $\Pi$ of the body must vanish. That is,

$$
\delta \Pi \equiv \delta U+\delta V=0,
$$

where $\delta U$ is the variation of total strain energy of the body as follows:

$$
\delta U=\int_{-a}^{a} \int_{-h / 2}^{h / 2} \int_{-\pi}^{\pi}\left(\sigma_{x} \delta \varepsilon_{x}+\sigma_{\theta} \delta \varepsilon_{\theta}+\sigma_{z} \delta \varepsilon_{z}+\sigma_{\theta z} \delta \gamma_{\theta z}+\sigma_{x z} \delta \gamma_{x z}+\sigma_{x \theta} \delta \gamma_{x \theta}\right) d \theta d z d x
$$

In (9), $V$ is negative of the work done on the body by the specified external forces. Here, $V=-2 F_{0} \cdot u_{1}(x= \pm a, \theta, z)=$ $-2 F_{0} C_{6} a$ and, therefore, $\partial V=-2 F_{0} \partial C_{6} a$. Also, the variations of strains in (11) are found as

$$
\begin{aligned}
\delta \varepsilon_{x} & =\delta C_{5}(R+z) \cos \theta+\delta C_{6}, \\
\delta \varepsilon_{\theta} & =\left(\delta V_{k}^{\prime}+\delta W_{k}\right) \frac{\Phi_{k}}{R}, \\
\delta \varepsilon_{z} & =\delta W_{k} \Phi_{k}^{\prime}, \\
\delta \gamma_{\theta z} & =\left(\delta W_{k}^{\prime}-\delta V_{k}\right) \frac{\Phi_{k}}{R}+\delta V_{k} \Phi_{k}^{\prime}, \\
\delta \gamma_{x z} & =\delta U_{k} \Phi_{k}^{\prime}, \\
\delta \gamma_{x \theta} & =-\delta C_{3}(R+z)+\delta U_{k}^{\prime} \frac{\Phi_{k}}{R} .
\end{aligned}
$$

Employing the fundamental lemma of calculus of variations the equilibrium equations and the associated boundary conditions of a laminate under tension are obtained as

$$
\begin{array}{r}
\delta U_{k}: Q_{x}^{k}-\frac{1}{R} \frac{d M_{x \theta}^{k}}{d \theta}=0, \\
\delta V_{k}: Q_{\theta}^{k}-\frac{1}{R}\left(\frac{d M_{\theta}^{k}}{d \theta}+R_{\theta}^{k}\right)=0, \\
\delta W_{k}: \frac{1}{R}\left(M_{\theta}^{k}-\frac{d R_{\theta}^{k}}{d \theta}\right)+N_{z}^{k}=0,
\end{array}
$$

$$
\begin{aligned}
\delta C_{3}: \int_{-\pi}^{\pi} \int_{-h / 2}^{h / 2} \sigma_{x \theta}(R+z) d z d \theta & =0, \\
\delta C_{5}: \int_{-\pi}^{\pi} \int_{-h / 2}^{h / 2} \sigma_{x}(R+z) \cos \theta d z d \theta & =0, \\
\delta C_{6}: \int_{-\pi}^{\pi} \int_{-h / 2}^{h / 2} R \sigma_{x} d z d \theta & =F_{0},
\end{aligned}
$$

where $F_{0}$ indicates the prescribed value of axial force applied at the two ends of the nanotube. Also, the following tractionfree boundary conditions must be satisfied:

$$
R_{\theta}^{k}=Q_{x}^{k}=N_{z}^{k}=0 \quad\left(\text { at } z= \pm \frac{h}{2}\right)
$$

where the generalized stress and moment resultants are defined as

$$
\begin{array}{r}
\left(N_{z}^{k}, Q_{x}^{k}, Q_{\theta}^{k}\right)=\int_{-h / 2}^{h / 2}\left(\sigma_{z}, \sigma_{x z}, \sigma_{\theta z}\right) \Phi_{k}^{\prime} d z, \\
\left(M_{\theta}^{k}, M_{x \theta}^{k}, R_{\theta}^{k}\right)=\int_{-h / 2}^{h / 2}\left(\sigma_{\theta}, \sigma_{x \theta}, \sigma_{\theta z}\right) \Phi_{k} d z .
\end{array}
$$

It is to be noted that, in (15) and (16a) and (16b), the superscript $k$ refers to the $k$ th wall in the nanotube.

\section{Nonlocal Theory (Constitutive Relations)}

According to Eringen [7-9], the stress field at a point $x$ in an elastic continuum depends not only on the strain field at the point but also on strains at all other points of the body. Eringen applied this fact to the atomic theory of lattice dynamics and experimental observations on phonon 
dispersion. The integral constitutive relations in an equivalent differential form are presented based on Eringen [7-9] as

$$
\left(1-\mu \nabla^{2}\right) \sigma_{i j}=C_{i j m n} \varepsilon_{m n}
$$

where $\nabla^{2}$ and $\mu=\left(e_{0} a\right)^{2}$ are the Laplacian operator and nonlocal parameter, respectively. In addition, $e_{0}$ and $a$ are a material constant and internal characteristic length, respectively. From both consideration of (17) and Hook's law, it is written that

$$
\left(1-\mu \nabla^{2}\right)\left(\begin{array}{c}
\sigma_{x}^{n l} \\
\sigma_{\theta}^{n l} \\
\sigma_{z}^{n l} \\
\sigma_{\theta z}^{n l} \\
\sigma_{x z}^{n l} \\
\sigma_{x \theta}^{n l}
\end{array}\right)^{(k)}
$$

$$
=\left[\begin{array}{cccccc}
\bar{C}_{11} & \bar{C}_{12} & \bar{C}_{13} & 0 & 0 & \bar{C}_{16} \\
\bar{C}_{12} & \bar{C}_{22} & \bar{C}_{23} & 0 & 0 & \bar{C}_{26} \\
\bar{C}_{13} & \bar{C}_{23} & \bar{C}_{33} & 0 & 0 & \bar{C}_{36} \\
0 & 0 & 0 & \bar{C}_{44} & \bar{C}_{45} & 0 \\
0 & 0 & 0 & \bar{C}_{45} & \bar{C}_{55} & 0 \\
\bar{C}_{16} & \bar{C}_{26} & \bar{C}_{36} & 0 & 0 & \bar{C}_{66}
\end{array}\right]^{(k)}\left(\begin{array}{c}
\varepsilon_{x} \\
\varepsilon_{\theta} \\
\varepsilon_{z} \\
\gamma_{\theta z} \\
\gamma_{x z} \\
\gamma_{x \theta}
\end{array}\right)^{(}
$$

where $\bar{C}_{i j}^{(k)}$ represent the off-axis stiffnesses. Multiplying the relation (18) by $\Phi_{k}^{\prime}$ and $\Phi_{k}$ and integrating them yield

$$
\begin{aligned}
& \int_{-h / 2}^{h / 2}\left\{\begin{array}{c}
\sigma_{\theta} \\
\sigma_{\theta z} \\
\sigma_{x \theta}
\end{array}\right\}^{(k)} \Phi_{k} d z-\mu \nabla^{2} \int_{-h / 2}^{h / 2}\left\{\begin{array}{c}
\sigma_{\theta} \\
\sigma_{\theta z} \\
\sigma_{x \theta}
\end{array}\right\}^{(k)} \Phi_{k} d z \\
& =\left[\begin{array}{cccccc}
\bar{C}_{12} & \bar{C}_{22} & \bar{C}_{23} & 0 & 0 & \bar{C}_{26} \\
0 & 0 & 0 & \bar{C}_{44} & \bar{C}_{45} & 0 \\
\bar{C}_{16} & \bar{C}_{26} & \bar{C}_{36} & 0 & 0 & \bar{C}_{66}
\end{array}\right]^{(k)} \\
& \int_{-h / 2}^{h / 2}\left(\begin{array}{c}
\varepsilon_{x} \\
\varepsilon_{\theta} \\
\varepsilon_{z} \\
\gamma_{\theta z} \\
\gamma_{x z} \\
\gamma_{x \theta}
\end{array}\right) \Phi_{k} d z
\end{aligned}
$$

$$
\begin{aligned}
& \int_{-h / 2}^{h / 2}\left\{\begin{array}{c}
\sigma_{z} \\
\sigma_{\theta z} \\
\sigma_{x z}
\end{array}\right\}^{(k)} \Phi_{k}^{\prime} d z-\mu \nabla^{2} \int_{-h / 2}^{h / 2}\left\{\begin{array}{c}
\sigma_{z} \\
\sigma_{\theta z} \\
\sigma_{x z}
\end{array}\right\}^{(k)} \Phi_{k}^{\prime} d z \\
& =\left[\begin{array}{cccccc}
\bar{C}_{13} & \bar{C}_{23} & \bar{C}_{33} & 0 & 0 & \bar{C}_{36} \\
0 & 0 & 0 & \bar{C}_{44} & \bar{C}_{45} & 0 \\
0 & 0 & 0 & \bar{C}_{44} & \bar{C}_{45} & 0
\end{array}\right] \\
& \cdot \int_{-h / 2}^{h / 2}\left(\begin{array}{c}
\varepsilon_{x} \\
\varepsilon_{\theta} \\
\varepsilon_{z} \\
\gamma_{\theta z} \\
\gamma_{x z} \\
\gamma_{x \theta}
\end{array}\right) \Phi_{k}^{\prime} d z .
\end{aligned}
$$

By considering (16a) and (16b) and strain displacement relations (Equation (9)), the stress resultants are given as

$$
\begin{aligned}
(1- & \left.\mu \nabla^{2}\right)\left(N_{z}^{k}, M_{\theta}^{k}, M_{x \theta}^{k}\right) \\
= & \frac{\left(B_{36}^{j k}, D_{26}^{k j}, D_{66}^{k j}\right) U_{j}^{\prime}}{R}+\frac{\left(B_{23}^{j k}, D_{22}^{k j}, D_{26}^{k j}\right) V_{j}^{\prime}}{R} \\
& +\left(A_{33}^{k j}, B_{23}^{k j}, B_{36}^{k j}\right) W_{j}+\frac{\left(B_{23}^{j k}, D_{22}^{k j}, D_{26}^{k j}\right) W_{j}}{R} \\
& -\left(A_{36}^{k}, B_{26}^{k}, B_{66}^{k}\right) R C_{3}-\left(\bar{B}_{36}^{k}, D_{26}^{k}, D_{66}^{k}\right) C_{3} \\
& +\left(A_{13}^{k}, B_{12}^{k}, B_{16}^{k}\right) R C_{5} \cos \theta \\
& +\left(\bar{B}_{13}^{k}, D_{12}^{k}, D_{16}^{k}\right) C_{5} \cos \theta \\
& -\left(A_{13}^{k}, B_{12}^{k}, B_{16}^{k}\right) C_{6}, \\
(1- & \left.\mu \nabla^{2}\right)\left(Q_{x}^{k}, Q_{\theta}^{k}, R_{\theta}^{k}\right) \\
= & \left(A_{55}^{k j}, A_{45}^{k j}, B_{45}^{k j}\right) U_{j}+\left(A_{45}^{k j}, A_{44}^{k j}, B_{44}^{k j}\right) V_{j} \\
& \quad \frac{\left(B_{45}^{j k}, B_{44}^{j k}, D_{44}^{k j}\right) V_{j}}{R}+\frac{\left(B_{45}^{j k}, B_{44}^{j k}, D_{44}^{k j}\right) W_{j}^{\prime}}{R},
\end{aligned}
$$

where the wall rigidities in (20) are defined as

$$
\begin{aligned}
& \left(A_{p q}^{k j}, \bar{B}_{p q}^{k j}, D_{p q}^{k j}\right)=\sum_{i=1}^{N} \int_{z_{i}}^{z_{i+1}} \bar{C}_{p q}^{(i)}\left(\phi_{k}^{\prime} \phi_{j}^{\prime}, \phi_{k} \phi_{j}^{\prime}, \phi_{k} \phi_{j}\right) d z, \\
& \left(A_{p q}^{k}, B_{p q}^{k}, \bar{B}_{p q}^{k}, D_{p q}^{k}\right) \\
& =\sum_{i=1}^{N} \int_{z_{i}}^{z_{i+1}} \bar{C}_{p q}^{(i)}\left(\phi_{k}^{\prime}, \phi_{k}, \phi_{k}^{\prime} z, \phi_{k} z\right) d z, \\
& (k, j=1,2, \ldots, N+1) .
\end{aligned}
$$


The nonlocal displacement equilibrium equations within LWT are obtained by substituting (20) into (13a), (13b), and (13c):

$$
\begin{aligned}
& \delta U_{k}: \frac{D_{66}^{k j}}{R^{2}\left(1-\left(\lambda^{2} / z^{2}\right) \mu\right)} U_{j}^{\prime \prime}-\frac{A_{55}^{k j}}{\left(1-\left(\lambda^{2} / z^{2}\right) \mu\right)} U_{j} \\
& +\frac{D_{26}^{k j}}{R^{2}\left(1-\left(\lambda^{2} / z^{2}\right) \mu\right)} V_{j}^{\prime \prime}-\frac{A_{45}^{k j}-B_{45}^{j k} / R}{\left(1-\left(\lambda^{2} / z^{2}\right) \mu\right)} V_{j} \\
& +\frac{\left(D_{26}^{k j} / R^{2}+\left(B_{36}^{k j}-B_{45}^{j k}\right) / R\right)}{\left(1-\left(\lambda^{2} / z^{2}\right) \mu\right)} W_{j}^{\prime} \\
& =\frac{B_{16}^{k}+D_{16}^{k} / R}{\left(1+\mu / z^{2}\right)} C_{5} \sin \theta \\
& +\frac{z_{k}}{R \mu^{0.5}}\left(e^{-\left(z_{k} / \mu^{0.5}\right) \theta}-e^{\left(z_{k} / \mu^{0.5}\right) \theta}\right), \\
& \delta V_{k}: \frac{D_{26}^{k j}}{R^{2}\left(1-\left(\lambda^{2} / z^{2}\right) \mu\right)} U_{j}^{\prime \prime}-\frac{\left(A_{45}^{k j}-B_{45}^{k j} / R\right)}{\left(1-\left(\lambda^{2} / z^{2}\right) \mu\right)} U_{j} \\
& +\frac{D_{22}^{k j}}{R^{2}\left(1-\left(\lambda^{2} / z^{2}\right) \mu\right)} V_{j}^{\prime \prime} \\
& -\frac{\left(A_{44}^{k j}-\left(B_{44}^{k j}+B_{44}^{j k}\right) / R+D_{44}^{k j} / R^{2}\right)}{\left(1-\left(\lambda^{2} / z^{2}\right) \mu\right)} V_{j} \\
& +\frac{\left(\left(B_{23}^{k j}-B_{44}^{j k}\right) / R+\left(D_{44}^{k j}+D_{22}^{k j}\right) / R^{2}\right)}{\left(1-\left(\lambda^{2} / z^{2}\right) \mu\right)} W_{j}^{\prime} \\
& =\frac{B_{12}^{k}+D_{12}^{k} / R}{\left(1+\mu / z^{2}\right)} C_{5} \sin \theta \text {, } \\
& \delta W_{k}: \frac{\left(\left(B_{45}^{k j}-B_{36}^{j k}\right) / R-D_{26}^{k j} / R^{2}\right)}{\left(1-\left(\lambda^{2} / z^{2}\right) \mu\right)} U_{j}^{\prime} \\
& +\frac{\left(\left(B_{44}^{k j}-B_{23}^{j k}\right) / R-\left(D_{44}^{k j}+D_{22}^{k j}\right) / R^{2}\right)}{\left(1-\left(\lambda^{2} / z^{2}\right) \mu\right)} V_{j}^{\prime} \\
& +\frac{D_{44}^{k j}}{R^{2}\left(1-\left(\lambda^{2} / z^{2}\right) \mu\right)} W_{j}^{\prime \prime} \\
& +\frac{\left(D_{22}^{k j} / R^{2}+\left(B_{23}^{j k}+B_{23}^{k j}\right) / R+A_{33}^{k j}\right)}{\left(1-\left(\lambda^{2} / z^{2}\right) \mu\right)} W_{j} \\
& =-\left(\bar{B}_{36}^{k}+B_{26}^{k}+R A_{36}^{k}+\frac{D_{26}^{k}}{R}\right) C_{3} \\
& +\left(A_{13}^{k}+\frac{B_{12}^{k}}{R}\right) C_{6} \\
& +\frac{\left(\bar{B}_{13}^{k}+B_{12}^{k}+R A_{13}^{k}+D_{12}^{k} / R\right)}{\left(1+\mu / z^{2}\right)} C_{5} \cos \theta
\end{aligned}
$$

$$
\begin{array}{r}
+\frac{1}{R}\left(e^{-\left(z_{k} / \mu^{0.5}\right) \theta}+e^{\left(z_{k} / \mu^{0.5}\right) \theta}\right) \\
k=1,2, \ldots, N+1 .
\end{array}
$$

Also, the global equilibrium equations of the nanotube are expressed in terms of displacement functions by substituting (9) into (19a), (19b) and the subsequent results into (14a), (14b), and (14c).

\section{Analytical Solution for Nanotube Structure}

The system in (22a), (22b), and (22c) shows $3(N+1)$ coupled ordinary differential equations with constant coefficients which may be displayed in a matrix form as

$$
[M]\left\{\eta^{\prime \prime}\right\}+[K]\{\eta\}=\left\{F_{1}\right\}\{C\}+\left\{F_{2}\right\},
$$

where

$$
\begin{aligned}
\{\eta\} & =\left\{\{U\}^{T},\{V\}^{T},\{\bar{W}\}^{T}\right\}^{T}, \\
\{U\} & =\left\{U_{1}, U_{2}, \ldots, U_{N+1}\right\}^{T}, \\
\{C\} & =\left\{C_{3}, C_{5}, C_{6}\right\}^{T}, \\
\{V\} & =\left\{V_{1}, V_{2}, \ldots, V_{N+1}\right\}^{T}, \\
\{\bar{W}\} & =\left\{\bar{W}_{1}, \bar{W}_{2}, \ldots, \bar{W}_{N+1}\right\}^{T}, \\
\bar{W}_{j} & =\int^{\theta} W_{j} d \theta .
\end{aligned}
$$

The coefficient matrices $[M],[K],\left[F_{1}\right]$, and $\left[F_{2}\right]$ in $(23)$ are defined in the Appendix. It can readily be confirmed that the general solution of (23) may be presented as

$$
\begin{aligned}
\{\eta\}= & {[\psi][\sinh (\lambda \theta)]\{k\}+[K]^{-1}\left\{F_{1}\right\}\{C\} } \\
& +[K]^{-1}\left\{F_{2}\right\}
\end{aligned}
$$

and $[\sinh (\lambda \theta)]$ is a $3(N+1) \times 3(N+1)$ diagonal matrix. That is,

$$
\begin{aligned}
& {[\sinh (\lambda \theta)]=\operatorname{diag}\left(\sinh \left(\lambda_{1} \theta\right), \sinh \left(\lambda_{2} \theta\right), \ldots,\right.} \\
& \left.\quad \sinh \left(\lambda_{3(N+1)} \theta\right)\right) .
\end{aligned}
$$

Also, $[\psi]$ and $\left(\lambda_{1}^{2}, \lambda_{2}^{2}, \ldots, \lambda_{3(N+1)}^{2}\right)$ are the model matrix and eigenvalues of $\left(-[M]^{-1}[K]\right)$, respectively. Matrix $\{k\}$ is an unknown vector representing $3(N+1)$ integration constants. The constants $C j(j=3,5,6)$ must be calculated within LWT analysis. Therefore, the boundary conditions in (15) are first imposed to find the vector $\{k\}$ in terms of the unknown parameters $C j(j=3,5,6)$. These constants are then calculated in terms of the specified axial force $F_{0}$ by satisfaction of the global equilibrium conditions in (14a), (14b), and (14c). 
TABLE 1: Mechanical properties of SWCNT, DWCNT, and MWCNT [26-28].

\begin{tabular}{lcc}
\hline Nanotubes & \multicolumn{2}{c}{ Mechanical properties } \\
& $E(\mathrm{TPa})$ & $v$ \\
\hline SWCNT & 1.80 & 0.19 \\
DWCNT & 1.33 & 0.19 \\
MWCNT & 0.95 & 0.19 \\
\hline
\end{tabular}

TABLE 2: Comparison of nondimensional displacement component in the longitudinal direction $\left(\bar{u}_{x}\right)$.

\begin{tabular}{ccccc}
\hline$L / h$ & $\mu$ & SWCNT & DWCNT & MWCNT $\left(\times 10^{1}\right)$ \\
\hline \multirow{4}{*}{100} & 0 & 1.082 & 1.276 & 2.170 \\
& 1.0 & 2.014 & 2.376 & 4.040 \\
& 2.0 & 2.052 & 2.421 & 4.116 \\
& 3.0 & 2.201 & 2.597 & 4.415 \\
& 4.0 & 2.657 & 3.135 & 5.329 \\
& 5.0 & 3.021 & 3.564 & 6.060 \\
\hline \multirow{4}{*}{200} & 0 & 1.136 & 1.340 & 2.279 \\
& 1.0 & 2.114 & 2.495 & 4.242 \\
& 2.0 & 2.154 & 2.542 & 4.322 \\
& 3.0 & 2.311 & 2.727 & 4.635 \\
& 4.0 & 2.789 & 3.292 & 5.596 \\
& 5.0 & 3.172 & 3.743 & 6.363 \\
\hline \multirow{5}{*}{500} & 0 & 1.150 & 1.358 & 2.308 \\
& 1.0 & 2.142 & 2.527 & 4.297 \\
& 2.0 & 2.182 & 2.575 & 4.378 \\
& 3.0 & 2.341 & 2.762 & 4.696 \\
& 4.0 & 2.826 & 3.334 & 5.669 \\
\hline
\end{tabular}

\section{Results and Discussion}

The numerical results are discussed for single-walled carbon nanotube (SWCNT), double-walled carbon nanotube (DWCNT), and multiwalled carbon nanotube (MWCNT) under extension. The mechanical properties of SWCNT, DWCNT, and MWCNT are given in Table 1 [26-28]. Furthermore, the displacement components are normalized as $\bar{u}_{i}=u_{i} / u_{0}$, where $u_{0}=L^{*} \sigma_{0} / E$ and $\sigma_{0}=\left(F_{0}\right) /\left(\pi / 4^{*}\left(O D^{2}-\right.\right.$ $\left.I D^{2}\right)$. An innermost radius of $8.5 \mathrm{~nm}$ is assumed for SWCNT, DWCNT, and MWCNT while assuming thickness of SWCNT $\left(h_{\text {eff }}\right)$ to be $0.34 \mathrm{~nm}$ [29]. Also, $L$ is the length of nanotubes. A 10-layer MWCNT is considered here in which $R /\left(N^{*} h_{\text {eff }}\right)=2 . L / h$ is defined as an aspect ratio.

The nanotube lengths, thickness, and nonlocal parameters play significant roles on determining the nondimensional displacement components. These parameters are studied numerically.

The numerical results for SWCNT, DWCNT, and MWCNT under axial force $\left(F_{0}\right)$ at the point in which $\theta=270^{\circ}$ are presented in Tables 2,3 , and 4 . Table 2 shows the effects of length, thickness, and nonlocal parameter $(\mu)$ on the nondimensional longitudinal displacement component $\left(\bar{u}_{x}\right)$. It is found from Table 2 that the longitudinal displacement
TABLE 3: Comparison of nondimensional displacement component in the circumferential direction $\left(\bar{u}_{\theta}\right)$.

\begin{tabular}{ccccc}
\hline$L / h$ & $\mu$ & SWCNT $\left(\times 10^{-2}\right)$ & DWCNT $\left(\times 10^{-2}\right)$ & MWCNT $\left(\times 10^{-1}\right)$ \\
\hline \multirow{6}{*}{100} & 1.110 & 1.309 & 2.226 \\
& 1.0 & 2.131 & 2.514 & 4.274 \\
& 2.0 & 2.265 & 2.672 & 4.543 \\
& 3.0 & 2.579 & 3.043 & 5.173 \\
& 4.0 & 3.001 & 3.540 & 6.018 \\
& 5.0 & 3.260 & 3.846 & 6.539 \\
\hline \multirow{6}{*}{200} & 0 & 1.165 & 1.375 & 2.337 \\
& 1.0 & 2.237 & 2.640 & 4.488 \\
& 2.0 & 2.378 & 2.806 & 4.770 \\
& 3.0 & 2.707 & 3.195 & 5.432 \\
& 4.0 & 3.150 & 3.717 & 6.318 \\
& 5.0 & 3.423 & 4.039 & 6.866 \\
\hline \multirow{6}{*}{500} & 0 & 1.180 & 1.393 & 2.368 \\
& 1.0 & 2.266 & 2.674 & 4.546 \\
& 2.0 & 2.409 & 2.842 & 4.832 \\
& 3.0 & 2.743 & 3.236 & 5.502 \\
& 5.0 & 3.190 & 3.765 & 6.401 \\
& 5.0 & 3.467 & 4.091 & 6.955 \\
\hline
\end{tabular}

TABLE 4: Comparison of nondimensional displacement component in the radial direction $\left(\bar{u}_{z}\right)$.

\begin{tabular}{ccccc}
\hline$L / h$ & $\mu$ & SWCNT $\left(\times 10^{-4}\right)$ & DWCNT $\left(\times 10^{-4}\right)$ & MWCNT $\left(\times 10^{-3}\right)$ \\
\hline \multirow{6}{*}{100} & 0 & 1.354 & 1.597 & 2.716 \\
& 1.0 & 1.887 & 2.226 & 3.785 \\
& 2.0 & 2.265 & 2.672 & 4.543 \\
& 3.0 & 2.402 & 2.834 & 4.818 \\
& 4.0 & 3.012 & 3.554 & 6.042 \\
& 5.0 & 3.223 & 3.803 & 6.465 \\
\hline \multirow{6}{*}{200} & 0 & 1.421 & 1.677 & 2.851 \\
& 1.0 & 1.981 & 2.337 & 3.974 \\
& 2.0 & 2.378 & 2.806 & 4.770 \\
& 4.0 & 2.522 & 2.976 & 5.059 \\
& 5.0 & 3.162 & 3.731 & 6.344 \\
\hline \multirow{5}{*}{500} & 3.384 & 3.993 & 6.788 \\
& 0 & 1.440 & 1.699 & 2.889 \\
& 1.0 & 2.0 & 2.368 & 4.026 \\
& 2.0 & 2.554 & 2.842 & 4.832 \\
4.0 & 3.203 & 3.014 & 5.125 \\
5.0 & 3.428 & 3.780 & 6.426 \\
\hline
\end{tabular}

component $\left(\bar{u}_{x}\right)$ increases with increasing the thickness while CNT length is kept constant. For SWCNT, the longitudinal displacement component $\left(\bar{u}_{x}\right)$ increases as the length of SWCNT increases. Observing Table 2, the longitudinal displacement component increases while the length and thickness of CNT are kept constant.

The effects of length, thickness, and nonlocal parameter $(\mu)$ on nondimensional circumferential displacement components $\left(\bar{u}_{\theta}\right)$ are studied in Table 3 . It is observed from 
Table 3 that the circumferential displacement component $\left(\bar{u}_{\theta}\right)$ increases with increasing the thickness while CNT length is kept constant. The circumferential displacement component $\left(\bar{u}_{\theta}\right)$ increases with increasing the length. Observing Table 3 , the circumferential displacement component $\left(\bar{u}_{\theta}\right)$ increases as the nonlocal parameter $(\mu)$ increases where length and thickness of CNT are fixed.

Table 4 reveals investigation for the effects of length, thickness, and nonlocal parameter $(\mu)$ on nondimensional radial displacement components $\left(\bar{u}_{z}\right)$. Table 4 shows the radial displacement component $\left(\bar{u}_{z}\right)$ increases as the thickness of nanotube increases. For the case of constant thickness, the radial displacement component $\left(\bar{u}_{z}\right)$ increases as CNT length increases. It is clear from Table 4 that the increase of nonlocal parameter $(\mu)$ causes increase of the radial displacement component $\left(\bar{u}_{z}\right)$.

\section{Conclusions}

We have developed a new nonlocal method for nanotube structures subjected to tension. Equations of motion using layer-wise theory were derived based on Eringen's differential constitutive equations of nonlocal elasticity. Variational statements of the theory were also presented to facilitate direct development of the nonlocal displacement models of layerwise theory. The equations of motion were then analytically solved for nanotubes under axial force to bring out the effect of nonlocal parameter. All displacement components increase with the increase in the nonlocal parameters $\mu$. Remark that the displacement components increase with increasing the length and thickness of CNT. Also, it is observed that the displacement components for MWCNT and DWCNT are greater than those for SWCNT. The values for displacement component in the longitudinal direction $\left(\bar{u}_{x}\right)$ are greater than those for the displacement components in the circumferential $\left(\bar{u}_{\theta}\right)$ and radial $\left(\bar{u}_{z}\right)$ directions for SWCNT, DWCNT, and MWCNT.

\section{Appendix}

The coefficient matrices $[M],[K],\left[F_{1}\right]$, and $\left[F_{2}\right]$ in $(23)$ are presented as

$$
\begin{gathered}
{[M]=\frac{1}{1-\left(\lambda^{2} / z^{2}\right) \mu}\left[\begin{array}{ccc}
\frac{1}{R^{2}}\left[D_{66}\right] & \frac{1}{R^{2}}\left[D_{26}\right] & \frac{1}{R}\left(\left[B_{36}\right]-\left[B_{45}\right]^{T}\right)+\frac{1}{R^{2}}\left[D_{26}\right] \\
\frac{1}{R^{2}}\left[D_{26}\right] & \frac{1}{R^{2}}\left[D_{22}\right] & \frac{1}{R}\left(\left[B_{23}\right]-\left[B_{44}\right]^{T}\right)+\frac{1}{R^{2}}\left(\left[D_{22}\right]+\left[D_{44}\right]\right) \\
{[0]} & {[0]} & \frac{1}{R^{2}}\left[D_{44}\right]
\end{array}\right],} \\
{[K]=\frac{-1}{1-\left(\lambda^{2} / z^{2}\right) \mu} \quad-\left[A_{45}\right]+\frac{1}{R}\left[B_{45}\right]^{T}} \\
\cdot\left[\begin{array}{cc}
-\left[A_{55}\right] & -\left[A_{44}\right]+\frac{1}{R}\left(\left[B_{44}\right]+\left[B_{44}\right]^{T}\right)-\frac{1}{R^{2}}\left[D_{22}\right] \\
-\left[A_{45}\right]+\frac{1}{R}\left[B_{45}\right] & \frac{1}{R}\left(\left[B_{44}\right]-\left[B_{23}\right]^{T}\right)-\frac{1}{R^{2}}\left(\left[D_{22}\right]+\left[D_{44}\right]\right)-\left[A_{33}\right]-\frac{1}{R}\left(\left[B_{23}\right]+\left[B_{23}\right]^{T}\right)-\frac{1}{R^{2}}\left[D_{22}\right]
\end{array}\right]
\end{gathered}
$$

$\left\{F_{1}\right\}$

$$
\begin{aligned}
& =\left\{\begin{array}{ccc}
\{0\} & \frac{\sin \theta}{1+\mu / z^{2}}\left(\left\{B_{16}\right\}+\frac{1}{R}\left\{D_{16}\right\}\right) & \{0\} \\
\{0\} & \frac{\sin \theta}{1+\mu / z^{2}}\left(\left\{B_{12}\right\}+\frac{1}{R}\left\{D_{12}\right\}\right) & \{0\} \\
-\left(\left\{\bar{B}_{36}\right\}+\left\{B_{26}\right\}+R\left\{A_{36}\right\}+\frac{1}{R}\left\{D_{26}\right\}\right) \theta & \frac{\sin \theta}{1+\mu / z^{2}}\left(\left\{\bar{B}_{13}\right\}+\left\{B_{12}\right\}+R\left\{A_{13}\right\}+\frac{1}{R}\left\{D_{12}\right\}\right)\left(\left\{A_{13}\right\}+\frac{1}{R}\left\{B_{12}\right\}\right) \theta
\end{array}\right\}, \\
& \left\{F_{2}\right\}=\left\{\begin{array}{c}
\frac{z_{k}}{R \mu^{0.5}}\left(e^{-\left(z_{k} / \mu^{0.5}\right) \theta}-e^{\left(z_{k} / \mu^{0.5}\right) \theta}\right) \\
\{0\} \\
\frac{\mu^{0.5}}{R z_{k}}\left(e^{\left(z_{k} / \mu^{0.5}\right) \theta}-e^{-\left(z_{k} / \mu^{0.5}\right) \theta}\right)
\end{array}\right\} .
\end{aligned}
$$




\section{Conflict of Interests}

The authors declare that there is no conflict of interests regarding the publication of this paper.

\section{References}

[1] A. Naghashpour and S. V. Hoa, "A technique for real-time detecting, locating, and quantifying damage in large polymer composite structures made of carbon fibers and carbon nanotube networks," Journal of Structural Health Monitoring, vol. 14, no. 1, pp. 34-45, 2015.

[2] A. Naghashpour and S. Van Hoa, "A technique for real-time detection, location and quantification of damage in large polymer composite structures made of electrically non-conductive fibers and carbon nanotube networks," Journal of Nanotechnology, vol. 24, no. 45, article 455502, Article ID 455502, 2013.

[3] H. Y. Sarvestani and A. Naghashpour, "Investigation of throughthickness stresses in composite laminates using layerwise theory," International Journal of Engineering Mathematics, vol. 2013, Article ID 676743, 11 pages, 2013.

[4] H. Y. Sarvestani, A. Naghashpour, and M. Heidari-Rarani, "Prediction of interlaminar stresses of an unsymmetric crossply laminate using layerwise and higher-order equivalent single-layer theories," International Journal of Aerospace and Lightweight Structures, vol. 3, no. 4, pp. 419-444, 2013.

[5] K. Behfar and R. Naghdabadi, "Nanoscale vibrational analysis of a multi-layered graphene sheet embedded in an elastic medium," Composites Science and Technology, vol. 65, no. 7-8, pp. 1159-1164, 2005.

[6] K. M. Liew, X. Q. He, and S. Kitipornchai, "Predicting nanovibration of multi-layered graphene sheets embedded in an elastic matrix," Acta Materialia, vol. 54, no. 16, pp. 4229-4236, 2006.

[7] A. C. Eringen, "Nonlocal polar elastic continua," International Journal of Engineering Science, vol. 10, pp. 1-16, 1972.

[8] A. C. Eringen, Continuum Physics, Volume IV: Polar and Nonlocal Field Theories, Academic Press, New York, NY, USA, 1976.

[9] A. C. Eringen, "On differential equations of nonlocal elasticity and solutions of screw dislocation and surface waves," Journal of Applied Physics, vol. 54, no. 9, pp. 4703-4710, 1983.

[10] A. C. Eringen and D. G. B. Edelen, "On nonlocal elasticity," International Journal of Engineering Science, vol. 10, pp. 233-248, 1972.

[11] A. C. Eringen and B. S. Kim, "Stress concentration at the tip of crack," Mechanics Research Communications, vol. 1, no. 4, pp. 233-237, 1974.

[12] S. J. Zhou and Z. Q. Li, "Length scales in the static and dynamic torsion of a circular cylindrical micro-bar," Journal of Shandong University of Technology, vol. 31, pp. 401-407, 2001.

[13] B. Akgöz and Ö. Civalek, "Application of strain gradient elasticity theory for buckling analysis of protein microtubules," Current Applied Physics, vol. 11, no. 5, pp. 1133-1138, 2011.

[14] F. Yang, A. C. M. Chong, D. C. C. Lam, and P. Tong, "Couple stress based strain gradient theory for elasticity," International Journal of Solids and Structures, vol. 39, no. 10, pp. 2731-2743, 2002.

[15] B. Akgöz and Ö. Civalek, "Free vibration analysis for singlelayered graphene sheets in an elastic matrix via modified couple stress theory," Materials \& Design, vol. 42, pp. 164-171, 2012.

[16] L. J. Sudak, "Column buckling of multiwalled carbon nanotubes using nonlocal continuum mechanics," Journal of Applied Physics, vol. 94, no. 11, pp. 7281-7287, 2003.
[17] J. N. Reddy and S. D. Pang, "Nonlocal continuum theories of beams for the analysis of carbon nanotubes," Journal of Applied Physics, vol. 103, no. 2, Article ID 023511, 2008.

[18] H. Heireche, A. Tounsi, A. Benzair, M. Maachou, and E. A. Adda Bedia, "Sound wave propagation in single-walled carbon nanotubes using nonlocal elasticity," Physica E, vol. 40, no. 8, pp. 2791-2799, 2008.

[19] M. Aydogdu, "Axial vibration of the nanorods with the nonlocal continuum rod model," Physica E: Low-Dimensional Systems and Nanostructures, vol. 41, no. 5, pp. 861-864, 2009.

[20] A. Farajpour, M. Mohammadi, A. R. Shahidi, and M. Mahzoon, "Axisymmetric buckling of the circular graphene sheets with the nonlocal continuum plate model," Physica E: Low-Dimensional Systems and Nanostructures, vol. 43, no. 10, pp. 1820-1825, 2011.

[21] M. Mohammadi, M. Ghayour, and A. Farajpour, "Free transverse vibration analysis of circular and annular graphene sheets with various boundary conditions using the nonlocal continuum plate model," Composites Part B: Engineering, vol. 45, no. 1, pp. 32-42, 2013.

[22] J. N. Reddy, "Nonlocal theories for bending, buckling and vibration of beams," International Journal of Engineering Science, vol. 45, no. 2-8, pp. 288-307, 2007.

[23] M. Levinson, "A new rectangular beam theory," Journal of Sound and Vibration, vol. 74, no. 1, pp. 81-87, 1981.

[24] J. N. Reddy, "Simple higher-order theory for laminated composite plates," Transactions ASME, Journal of Applied Mechanics, vol. 51, no. 4, pp. 745-752, 1984.

[25] G. Alotta, G. Failla, and M. Zingales, "Finite element method for a nonlocal Timoshenko beam model," Finite Elements in Analysis and Design, vol. 89, pp. 77-92, 2014.

[26] M. M. J. Treacy, T. W. Ebbesen, and J. M. Gibson, "Exceptionally high Young's modulus observed for individual carbon nanotubes," Nature, vol. 381, no. 6584, pp. 678-680, 1996.

[27] X. Wei, Q. Chen, L.-M. Peng, R. Cui, and Y. Li, “Tensile loading of double-walled and triple-walled carbon nanotubes and their mechanical properties," Journal of Physical Chemistry C, vol. 113, no. 39, pp. 17002-17005, 2009.

[28] M.-F. Yu, O. Lourie, M. J. Dyer, K. Moloni, T. F. Kelly, and R. S. Ruoff, "Strength and breaking mechanism of multiwalled carbon nanotubes under tensile load," Science, vol. 287, no. 5453, pp. 637-640, 2000.

[29] X. Wang and H. K. Yang, "Bending stability of multiwalled carbon nanotubes," Physical Review B, vol. 73, no. 8, Article ID 085409, 2006 

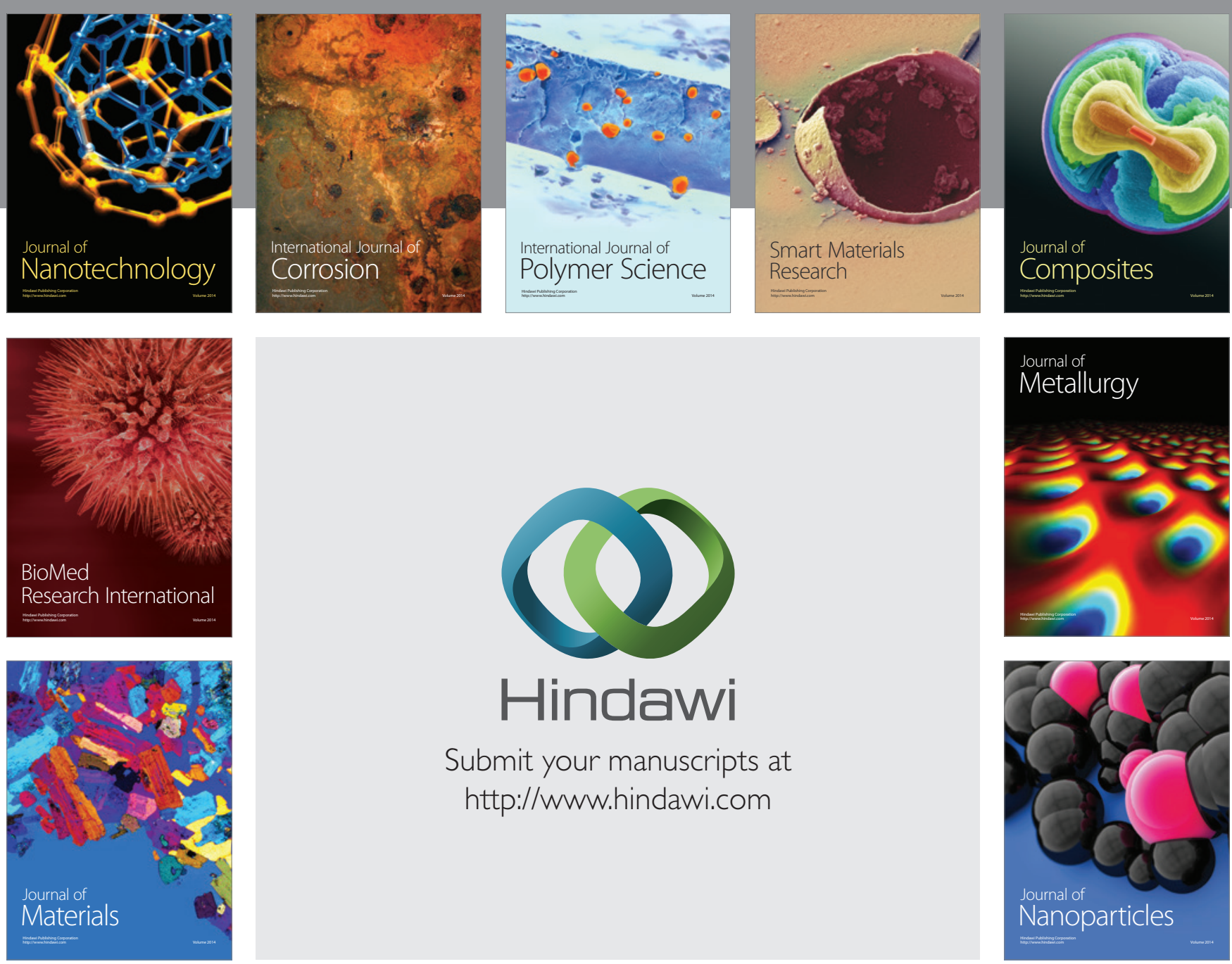

Submit your manuscripts at http://www.hindawi.com
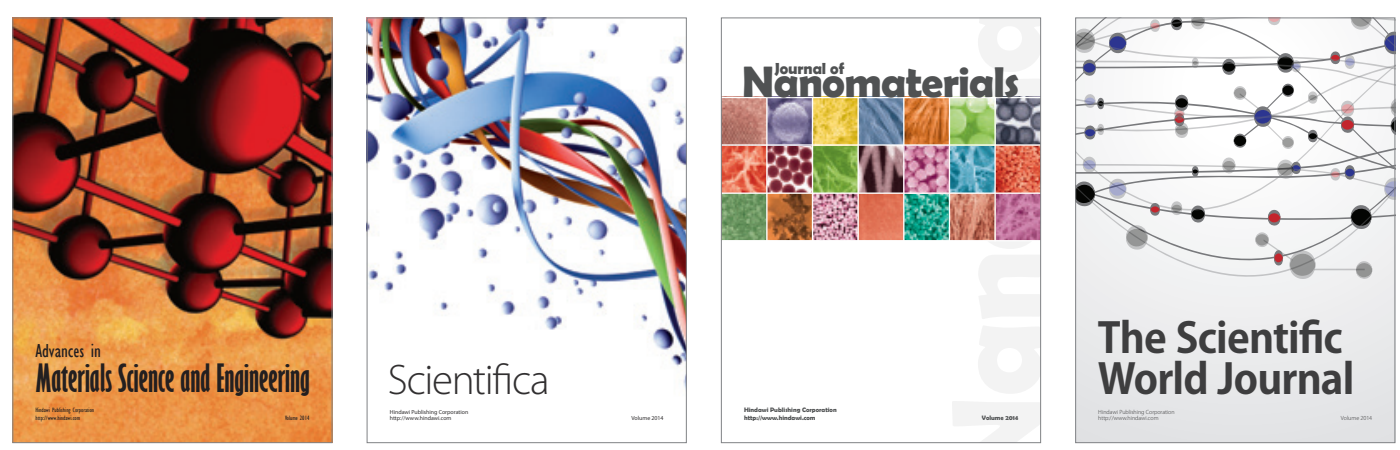

\section{The Scientific World Journal}
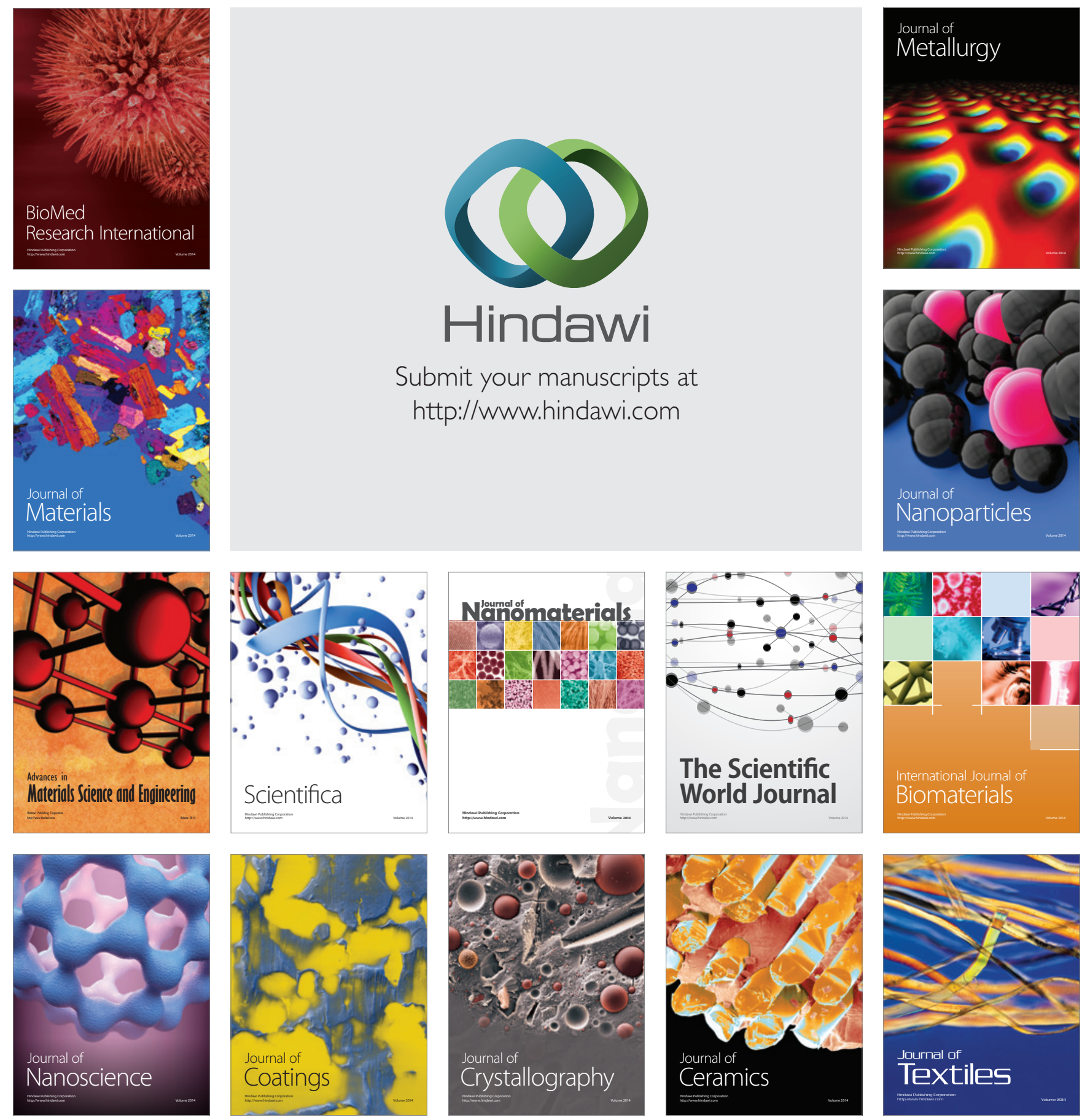